RESEARCH NOTE

\section{Overdiagnosis of Intestinal Amoebiasis Associated to Serial Microscopical Examination of Faeces. Some Precisions on a Problem}

\author{
Luis Fonte ${ }^{+}$, Ana M Montalvo, \\ Esteban Alberti, Fidel Núñez, \\ Lázara Rojas
}

Instituto de Medicina Tropical "Pedro Kourí”, Autopista Novia del Mediodía, km 61/2, Apartado Postal 601, Marianao 13, Ciudad de la Habana, Cuba

\section{Key words: Entamoeba histolytica - intestinal amoebiasis - overdiagnosis - microscopical examination - ENZYMEBA}

In spite of the accumulated evidences favoring the existence of Entamoeba histolytica as a complex of two species morphologically identical, but with different pathogenecity, Entamoeba histolytica, Schaudinn, 1903, and Entamoeba dispar, Brumpt, 1925 (LS Diamond \& CG Clark 1993 J Euk Microbiol 40: 340-344), the microscopical examination of faeces continuous to be the most widely used laboratory method for the diagnosis of intestinal amoebiasis.

This procedure has two additional limitations: since the excretion of the cyst of E. histolytica is intermittent, it requires the examination of at least three specimens on separate days to minimize the presence of false negatives (JA Walsh 1986 Rev Infect Dis 8: 228-238). The differentiation of $E$. histolytica from other cells in faeces is tedious and frequently leukocytes in the stool are mistakenly identified as E. histolytica (F Anaya \& G Sabanero 1989 Trans $R$ Soc Trop Med Hyg 83: 210-213, DA Bruckner 1992 Clin Microbiol Rev 5: 356-369). These difficulties have been frequently mentioned but not rigorously estimated.

Recently, AL Luaces et al. (1992 Parasitology 105: 203-205, 1993 Parasitol Today 9: 69-71) developed a highly effective method for the detection of E. histolytica in faeces. The technique,

\footnotetext{
${ }^{+}$Corresponding author. Fax: +53-7-246051. E-mail: fonte@ipk.sld.cu

Received 4 February 1998

Accepted 22 June 1998
}

which they named ENZYMEBA, is based on immuno-enzymatic capture of histolysaine, a cysteine proteinase of $E$. histolytica first described by AL Luaces and AJ Barret (1988 Biochem J 250: 903-907). Taking into account the advantages of ENZYMEBA method, included its ability to perform effective diagnosis using frozen samples, we decided to determine with more precision the misdiagnosis of intestinal amoebiasis associated to the microscopical examination of faeces.

Three stool samples were collected from 288 individuals who visited the parasitology services of three hospitals of Havana City by different reasons, more frequently diarrhoeal diseases. All the specimens were studied by the routine procedures used for microscopical examination of faeces in the corresponding hospital.

To be assayed by ENZYMEBA, a portion of each sample was frozen at $-20^{\circ} \mathrm{C}$ and sent to the Parasitology Laboratory of the Tropical Medicine Institute "Pedro Kourí" (IPK) of Cuba. The ENZYMEBA test, a commercial enzyme immunoassay kit produced at IPK, was used according to the manufacturer's instructions, based on the procedure described by Luaces et al. (loc. cit.).

The results are shown in the Table. The three samples of 151 individuals were negative to $E$. histolytica by morphological observation. Of these persons, only the specimens of three $(1.9 \%)$ were positive by ENZYMEBA (as this technique is based on the detection of enzymatic activity of histolysaine, a positive result is possible in the absence of the intact parasite in the sample). This result, by using a different procedure, confirms with precision that the serial microscopical examination of at least three stool samples per individual satisfactorily reduces the risk of false negative diagnosis of intestinal amoebiasis.

However, as we can see in the Table, of the 137 persons whose three samples were at least once positive by microscopical observation, only the specimens of $28(21.9 \%)$ were positive by ENZYMEBA. The number of microscopical false positives was superior when the number of samples analyzed per individual was higher (55, 88 and 109 false positives after the first, second and third samples, respectively). The ENZYMEBA test has the advantage of not being dependent of the skill of a microscopist. The report of cases found positive by microscopical examination and negative by ENZYMEBA was first done by Luaces et al. (loc. cit.). Faeces of those individuals were cultivated in Robinson's medium in order to try to isolate $E$. histolytica, but from none of them was growth of parasites observed.

Taken these results together, we have objectively demonstrated that the current solution of the microscopical subdiagnosis of amoebiasis, the ex- 


\section{TABLE}

Results of microscopical examination and the

ENZYMEBA test on faecal samples of 288 individuals (three per each)

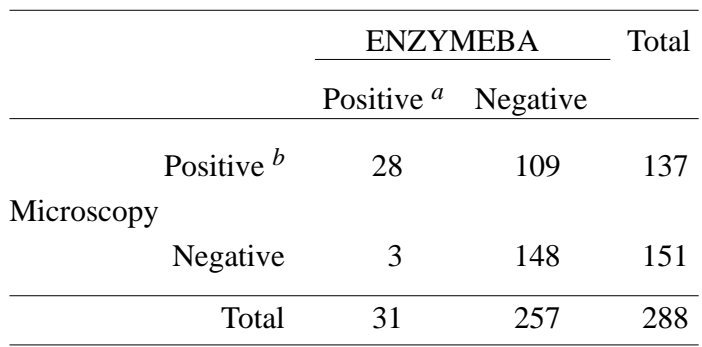

$a$ : ENZYMEBA test was positive in the three samples of 31 persons; $b$ : microscopical examination was considered positive when cysts and/or trophozoites of Entamoeba histolytica/E. dispar were observed in at least one sample per individual. amination of serial samples of faeces, leads to an increase of the occurrence of microscopical overdiagnosis of this infection. Thus, it is clear that there is an urgent need of complementary tests for an efficient diagnosis of amoebiasis. The new methods must be based on appropriate technologies for use in a broad range of logistic conditions, included those more frequently present at the health services of developing countries, and must differentiate between $E$. histolytica and E. dispar infections.

Stool microscopy, due to its low cost and capacity for detecting different parasitic protozoa and helminths in the same assay, will continue to play an essential role in supporting the physicians in the diagnosis of intestinal parasitism. Accordingly, the improvement of the technician's competence to execute the microscopical examination of faeces remains a necessary approach to the reduction of the overdiagnosis of intestinal amoebiasis. 